Finset, A., Stensrud, T.L., Holt, E., Verheul, W., Bensing, J. Electrodermal activity in response to empathic statements in clinical interviews with fibromyalgia patients. Patient Education and Counseling: 2011, 82(3), 355-360

\begin{tabular}{|l|l|}
\hline Postprint Version & 1.0 \\
\hline Journal website & $\underline{\text { http://dx.doi.org/10.1016/j.pec.2010.12.029 }}$ \\
\hline Pubmed link & $\underline{\text { http://www.ncbi.nlm.nih.gov/pubmed/21295937 }}$ \\
\hline DOI & $10.1016 /$ j.pec.2010.12.029 \\
\hline
\end{tabular}

This is a NIVEL certified Post Print, more info at http://www.nivel.eu

\title{
Electrodermal activity in response to empathic statements in clinical interviews with fibromyalgia patients
}

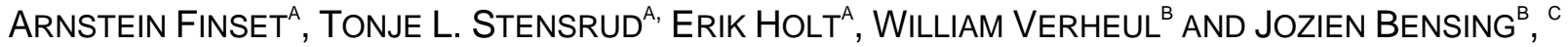 \\ ${ }^{a}$ Department of Behavioral Sciences in Medicine, Institute of Basic Medical Sciences, Faculty of Medicine, \\ University of Oslo, Oslo, Norway \\ ${ }^{\mathrm{b}}$ NIVEL (Netherlands Institute for Health Services Research), Utrecht, The Netherlands \\ ${ }^{\mathrm{c}}$ Faculty of Social and Behavioral Science, Utrecht University, Utrecht, The Netherlands
}

\begin{abstract}
Objective: To study patients' electrodermal response (1) to an interviewer's empathic statements vs. inattention to emotional concerns and (2) to the emotional content in clinical interviews.

Methods: Patients with fibromyalgia $(n=48)$ were given a clinical interview in a randomized $2 \times 2$ design, in which interviewer empathic or inattentive behavior (scenario A) and the emotional or neutral content of the interview (scenario B) were manipulated.

Electrodermal activity (EDA) was measured during the interview. The patients' affective state (PANAS) and patient satisfaction were also assessed.

Results: In scenario A a significant main effect of empathy was found, indicating an increased tonic EDA (skin conduction level, SCL) during the empathy condition $(\mathrm{p}<.05)$. In scenario B there was a main effect of empathy $(\mathrm{p}<.01)$ and a significant empathy $\times$ content interaction effect $(p<.01)$. There was a strong positive association between empathy and patient satisfaction $(\mathrm{p}<.0001)$.

Conclusions: Empathic statements by the interviewer were associated with increased SCL in patients, most so in interviews with emotional content.

Practice implications: Psychophysiological variables such as EDA may be applied in clinical communication research on emotional communication, but the limitations of such measures should also be recognized.
\end{abstract}

\section{INTRODUCTION}

Emotions are displayed in many medical consultations, and much attention has been given to how patients express emotions and how providers respond. Emotions are often presented as subtle cues to hidden emotions, but sometimes explicitly expressed as emotional concerns (for review, see [1]). Providers are often inattentive to these cues and concerns and overlook them or fail to respond [2], but cues and concerns may also be responded to with empathic responses. An empathic response may be defined as an explicit appreciation of the patient's emotions and expression of that awareness to the patient [3]. Empathy has been given much attention in clinical communication research (for review, see [4]).

Most studies on emotions in clinical communication research have applied psychological methods. However, during interpersonal interaction emotions may also elicit psychophysiological responses, such as 
Finset, A., Stensrud, T.L., Holt, E., Verheul, W., Bensing, J. Electrodermal activity in response to empathic statements in clinical interviews with fibromyalgia patients. Patient Education and Counseling: 2011, 82(3), 355-360

electrodermal activity (EDA). The study of physiological responses in interpersonal settings is sometimes referred to as sociophysiology, a term that also has been applied to studies of the provider-patient relationship [5].

EDA is one of the most widely studied response systems in psychophysiological research. The principle of EDA is to record electrical activity in the skin. As sweat fills the sweat ducts in the skin, skin conductance increases [6]. These increases in skin conductance may be phasic skin conductance responses, in terms of sudden bursts of electric activity in immediate response to discrete stimuli (skin conductance responses, $\mathrm{SCR}$, often referred to as spikes) or tonic changes in skin conductance level (SCL), i.e. gradual changes in the mean level of EDA over time. An advantage of EDA over some other physiological measures, such as heart rate, is the linear increase from before to after stimulation, rather than a curvilinear pattern with a post-stimulation decrease before subsequent increase seen in the cardiac response [7].

Human sweat glands receive predominantly sympathetic cholinergic innervations from fibers originating in the sympathetic chain, and EDA is thus considered to provide a relatively direct and undiluted representation of sympathetic activity, more so than most other psychophysiological parameters [8] and [9].

Sympathetic activity is viewed as a major component in emotional experience and emotional behavior [9], and electrodermal responses may therefore be viewed as inherent aspects of emotional behavior. The psychophysiological responses represent the somatic components in emotion, and EDA is a marker of sympathetic arousal which through the sympathetic-adrenal-medullary (SAM) system is associated with disease related processes, for instance in pain [10] and anxiety disorders [11]. With its strong association with the sympathetic activity EDA is therefore a physiological marker of emotion commonly applied in emotion research [9], also in studies on psychotherapy [12], [13] and [14] and clinical communication [15].

When patients tell about their illness and their concerns, clinicians have a number of different options on how to respond to the patients' explicit emotions or utterances with potential emotional salience [16]. Different provider responses to patient emotion may elicit different psychophysiological responses in patients. Although some physiological aspects of empathic communication have been studied [14], [15], [16] and [17], no study to our knowledge has explored the nature of psychophysiological responses of patients to inattention to emotions on the one hand and explicit empathic utterances from providers on the other.

In the present study we therefore investigated the electrodermal responses of arranged medical interviews where the interviewer systematically varied her responses to cues, concerns and worries expressed by the patients. The rationale for the study is that better knowledge of physiological responses to empathic statements could give better insight into the dynamics of emotion in medical interviews, with potential implications for the communication behavior of clinicians.

From a clinical point of view it would be particularly interesting to study physiological responses in patients that might be sensitive to how providers respond to their emotions. We therefore chose patients with the fibromyalgia syndrome (FMS) as informants in the present study. FMS is characterized by chronic widespread pain, fatigue, sleep disturbances and often also other symptoms and concerns [18]. It has been reported that FMS patients often experience their relations to doctors as difficult [19]. Accounts that fibromyalgia patients feel rejected or ignored, and "not taken seriously" are common [20]. Moreover, a number of studies have indicated problems in affective processing in many FMS patients, and enhanced defensive sympathetic nervous system activation patterns, which again could be related to enhanced symptomatology [21]. Given these characteristics of FMS patients it would be interesting to investigate their electrodermal responses to emotions in a medical interview.

We decided to investigate two scenarios, A and B.

In the A scenario the interviewer did not actively initiate emotional topics, but responded consistently either with statements acknowledging patients' concerns (empathy) or with avoiding to respond to patients' descriptions of subjective concerns (inattention to patient concern). Our hypothesis was that empathic statements would serve as a buffer against the potential stress of the medical interview. There is much evidence that positive social support may attenuate psychophysiological activation [22]. Given the need of FMS patients to be taken seriously one might expect that empathic acknowledgement might prevent an increase in electrodermal activation during the interview. On the other hand, we expected that avoiding to respond to potentially emotional concerns and a display of inattention to emotions on the part of the interviewer might provoke irritation and anger, which often have been associated with increased EDA level [9]. 
Finset, A., Stensrud, T.L., Holt, E., Verheul, W., Bensing, J. Electrodermal activity in response to empathic statements in clinical interviews with fibromyalgia patients. Patient Education and Counseling: 2011, 82(3) $355-360$

An alternative hypothesis for the A scenario would be that an empathic response would increase EDA activity. The findings on EDA responses to positive emotions expected as potential effects of empathic communication, such as affection, contentment and happiness are mixed in the literature, with reports both of increased, unchanged and (less often) decreased SCL [9]. Thus, positive emotions elicited by the interviewer might actually be associated with increased EDA activation.

In the B Scenario the interviewer continued an empathic or inattentive communication style, but asked either explicit questions about patient emotions or introduced a likely neutral theme (discussion of diet). We expected that patients would respond to the explicit emotional content and questions about emotional reactions from the interviewer with increased EDA response, but that a neutral topic would not elicit EDA changes.

\section{METHODS}

\subsection{Participants}

Patients with a confirmed fibromyalgia diagnosis were recruited through their attendance at a rehabilitation centre in Moss, Norway. Only female patients were included in the study. Patients admitted to the centre with sufficient proficiency in the Norwegian language were consecutively invited to participate in the study. Forty-eight patients were included in the study. The patients did not get any sort of payment for participating. The patients were randomly allocated to one of four experimental conditions (see below).

The interviewer was a researcher with a Master's degree in psychology (TLS). She was trained in executing the experimental conditions before starting data gathering.

\subsection{Procedures}

The patients were invited to participate in a study on provider-patient communication. The informed consent letter mentioned that doctor consultations might generally be experienced as stressful and that EDA level therefore would be measured throughout the interview. The patients were also informed that the interviewer would ask them some questions about their thoughts and feelings about their disease. They were informed that the interview would be videotaped.

The experiment took place at the rehabilitation centre in one of their meeting rooms. The room was arranged to look like a doctor's office. At the participating day the patient showed up at the meeting room and was greeted by a research technician who told the participant about the procedure, gave her the questionnaires and applied electrodes to measure EDA. After answering the questionnaires baseline measures were taken. Relaxing music was played for four minutes. The participant was told to relax with eyes closed. After baseline measures the interviewer was called in, and the interview was performed. After the interview the patient filled out questionnaires.

The interviewer was instructed to perform the interview according to four different conditions. Sealed envelopes with written instructions about which condition to follow were given her before each interview. She wore a white coat and was instructed to keep the interview in about two times ten minutes. The experimental conditions are described below.

After the interview the interviewer left and the research technician came back and debriefed the patient, letting her know that the interviewer was instructed in behaving in a certain way, and that the interviewer therefore might be experienced differently considered by different participants. At the same time they were given questionnaires to fill out.

\subsection{Experimental conditions}

The interview was divided into two parts, scenario A and scenario B:

Scenario A. Empathy vs. inattention to emotion.

In scenario A general questions about the illness and it's treatment were given. In the Empathy condition potentially emotional concerns of patients were responded to by the interviewer in an empathic way during the interview. Empathic responses and facilitating questions showing interest and empathy were given, such as "I understand", "That must have been difficult for you", "That sounds stressful", based on our definition of empathy as appreciation of the patient's emotions and expression of that awareness back to the patient. On the other hand, in the inattention to emotion condition the interviewer avoided to respond directly to emotional concerns, and responses to patients' statements were given with factual questions or not given at all. 
Finset, A., Stensrud, T.L., Holt, E., Verheul, W., Bensing, J. Electrodermal activity in response to empathic statements in clinical interviews with fibromyalgia patients. Patient Education and Counseling: 2011, 82(3) 355-360

Scenario B. Emotional vs. neutral content:

In the emotional content condition the interviewer continued an empathic or inattentive communication style, but asked explicit questions about patients' emotional reactions. The neutral content condition included questions about diet.

\subsection{Physiological measures}

\subsubsection{Electrodermal activity}

EDA activity was measured using the Biopac MP150 system which was connected to a Windows XP operated laptop running Acknowledge 3.8 (Biopac) data acquisition program. The Biopac GSR100C transducer module was used for exciting a $0.5 \mathrm{~V}$ constant current and 200 samples per second were recorded. Disposable gel finger electrodes (type: $\mathrm{Ag}-\mathrm{AgCl}$, contact area: $1 \mathrm{~cm}$ diameter) were placed on the second and third finger of the subject's non-dominant hand. The interview was recorded using a digital video camera connected to a second windows XP operated notebook computer and the video signal was directly fed into the Observer XT 7 program (Noldus). Both computers where connected and Acknowledge and Observer synchronized the video signal with the physiological data. A low pass filter was used to eliminate any high frequency noise components of the EDA signal (cut off frequency $1 \mathrm{~Hz}$, number of coefficients: 800).

Changes in tonic EDA were calculated by subtracting mean levels of skin conductance activity during baseline from mean levels of skin conductance during scenarios A and B respectively and will be referred to in the following as SCL (skin conductance level).

Changes in phasic EDA were calculated by counting the number of spikes (threshold level: $0.05 \mu$ Siemens) per minute during baseline and parts 1 and 2 and will be referred to as SCR (skin conductance responses).

\subsection{Questionnaires}

\subsubsection{Positive Affect Negative Affect Schedule (PANAS)}

Positive and negative affects were measured both before and after interview. It was measured with the PANAS which is a 20 item self-report questionnaire measuring positive and negative affect. PANAS has two times ten emotion words to be rated on a 5 point scale. It has been shown to possess adequate psychometric qualities [23].

\subsubsection{Patient satisfaction questionnaire}

The Patient Experience Questionnaire (PEQ) was administered after the consultation. PEQ is a 22 items questionnaire designed to assess immediate patient experiences after medical consultations [24].

\subsection{Statistics}

Associations between continuous variables were explored by computations of Pearson's product-moment correlations.

One- and two-way ANCOVAs were applied to investigate the effect of experimental conditions and baseline SCL, on SCL variance.

Analyses were performed applying the Statistical Package for Social Sciences (SPSS Inc., Chicago), SPSS for Windows version 16 .

\section{RESULTS}

SCL values rose significantly from Baseline to mean values during scenario A of the experiment $(t=6.6$; $\mathrm{p}<.001$ ) but remained at about the same level during scenarios A and B (Table 1). The number of EDA spikes per minute rose from .9 at Baseline to a mean of 3.4 during scenario $\mathrm{A}(\mathrm{t}=8.4 ; \mathrm{p}<.001)$, but did not increase significantly further during scenario B of the experiment (not shown in table).

\section{[TABLE 1]}

There was a significant reduction in negative affect from baseline to the end of the experiment (not shown in table). There were no significant correlations between psychological variables (PANAS and PEQ) and EDA values, neither in terms of SCL nor SCR. 
Finset, A., Stensrud, T.L., Holt, E., Verheul, W., Bensing, J. Electrodermal activity in response to empathic statements in clinical interviews with fibromyalgia patients. Patient Education and Counseling: 2011, 82(3) $355-360$

The experimental conditions produced no significant effects on phasic electrodermal arousal (SCR), and no significant interactions were found.

To test the effects of empathy vs. inattention to emotions in scenario A on SCL, we conducted a one-way ANCOVA with mean SCL during scenario A as dependent variable, and Empathy vs. Inattention as fixed factor, with baseline SCL as covariate. In the total sample we found a significant main effect of Empathy vs. Inattention $(\mathrm{F}=5.2, \mathrm{p}<.05)$ indicating a higher EDA level during the empathic than the inattention condition during scenario A of the experiment (Table 1 and Fig. 1).

\section{[FIGURE 1]}

In scenario B a second experimental condition was introduced, the Emotional vs. Neutral contents condition. We still found a main effect of empathy $(\mathrm{F}=7.9, \mathrm{p}<.01)$ and a significant interaction between the two experimental conditions (empathy/inattention $\mathrm{x}$ emotional content/neutral content) indicating more increase in SCL from baseline to scenario B of the experiment among patients in the combination of high empathy and emotional (as opposed to neutral) content $(\mathrm{F}=9.2, \mathrm{p}<.01)$ (Table 1 and Fig. 1).

There were no significant effects either of Empathy vs. Inattention or Emotional vs. Neutral content on positive or negative affect, but there was a strong significant positive effect of Empathy on Satisfaction $(\mathrm{F}=17.0, \mathrm{p}<.0001)($ Table 1 and Fig. 1$)$.

\section{DISCUSSION AND CONCLUSION}

\subsection{Discussion}

In this study of arranged consultations with patients with fibromyalgia we found, contrary to expectations, that an empathic communication style was associated with increased EDA level (SCL) even when the provider did not explicitly introduce an emotional content. The significant effect of empathy was even stronger when an emotional content was introduced by the interviewer in terms of explicit questions about emotional reactions.

Avoidance to respond to patient concerns (the Inattention to emotions condition) was associated with much lower SCL during the consultation than empathic responses were. Moreover, empathic communication style was also associated with more satisfaction than a communication style characterized by inattention to emotion. Finally, there were only marginal effects of communication style on positive and negative affect.

Our consistent finding of an increase in SCL in clinical interviews related to empathic statements from the interviewer but not to inattention to emotion is new and asks for reflection. The findings indicate that the dialogue in the empathy condition took on a more emotionally aroused quality, even if the interviewer responded to patient statements with empathic and supportive remarks, intended to buffer arousal.

Interestingly, the empathy condition was also strongly associated with increased patient satisfaction, even if SCL and satisfaction score were uncorrelated.

There may be different interpretations of the increased SCL in the empathy conditions of the experiment. One possible interpretation is that this SCL increase might have a positive valence, as empathy was also associated with higher patient satisfaction. Positive emotions have been associated with increased SCL, but the findings have not been consistent [9]. An opposite speculation would be that the empathic remark when presented functions to reinforce rather than buffer the distress.

However, the EDA response does not always correspond with the subjective experience. For instance, in the process of emotion regulation, subjective experience may be down-regulated, whereas the physiological response is up-regulated. Gross found that when subjects were instructed to withhold or suppress a conscious emotional response, they succeeded in reducing their facial expressions of emotions and subjective reports, but displayed instead an increase in psychophysiological responses [25]. In that case the physiological response may be considered as a marker of inhibited subjective expression of emotions. Thus, the increased SCL as a response to empathy observed in our study even before emotional content was explicitly introduced may therefore be interpreted as a vague and perhaps not consciously acknowledged resistance to emotional talk. We suggest that such a response could be part of a pattern of disturbed emotion regulation characterized by inhibited subjective experience and verbal expression seen in fibromyalgia and 
Finset, A., Stensrud, T.L., Holt, E., Verheul, W., Bensing, J. Electrodermal activity in response to empathic statements in clinical interviews with fibromyalgia patients. Patient Education and Counseling: 2011, 82(3) 355-360

increased SCL, potentially contributing to maintenance of sympathetic-adrenal-medullary (SAM) related pathology [26].

At this stage, we can only speculate about the validity of these interpretations, and much more research is needed on the impact of certain communication styles on patients' psychophysiological responses. But our findings illustrate the potential relevance for applying psychophysiology in the study of empathy in clinical communication research, here limited to the relevance of EDA.

Limitations. The present study has a number of limitations. Some of these limitations illustrate general limitations and precautions to be considered in studies of psychophysiological variables in clinical communication research.

First, the present study illustrates that the very strength of psychophysiological methods, tapping into aspects of emotions without subjective awareness, may at the same time represent a limitation. EDA methodology assesses the arousal component of emotion, whereas the subjective reports will reflect the quality or the valence of the emotional response. In this study we did not find any correlations between EDA measures and psychological variables. Yet, there is often more correspondence with EDA measures and subjective emotional report in psychophysiological experiments than was the case in our study [9]. Because of the lack of correlation with subjective experience it may be hard to judge whether the increased arousal is "good" or "bad". It is important not to uncritically consider increased EDA as a stress response, when it actually could be an indicator of positive emotion as well.

Second, we know that different psychophysiological measures may give different results. In our study we found effects on SCL, but not on phasic EDA responses. Preferably a number of different parameters should be investigated in order to achieve a broader assessment of psychophysiological aspects.

Third, the sample size was small and the study was not powered to investigate the impact of individual differences. This is a limitation, since differences in EDA have been reported between individuals with different personality characteristics. There is seldom a one-to-one relationship between EDA (and other psychophysiological response systems) and personality. Stemmler and Wacker have recently pointed out that the association between personality and physiology often is context dependent: "The individual appraisal of situations leads to interindividual differences in states which in turn may activate matching personality dispositions (p. 550)" [27]. Thus, interesting findings may be lost if relevant individual differences are not explored or at least controlled for. In future studies individual differences should be investigated.

Fourth, in the present study, all patients were suffering from fibromyalgia. It is difficult to judge to which extent the increased SCL response to empathy is a general phenomenon or specifically associated with illness related processes in this patient group. This means that the results cannot be generalized to patients with other types of health problems. In subsequent studies the EDA responses of FMS patients should be compared to healthy controls.

Fifth, the interviews in the present study were not real medical consultations, but arranged interviews between patients and a researcher. The study is, however, conducted in a real clinical setting with patients who took part in a rehabilitation program, but it may be difficult to generalize from this setting to real consultations.

Sixth, it is a limitation of our study that we only measure EDA in patients, not in the interviewer. Empathy should ideally be studied as a dyadic phenomenon.

Finally, only female subjects are included, prohibiting exploration of potential sex differences in EDA responses.

Research on electrodermal activity may employ methodological approaches not used in the present study. Two such lines of research shall be mentioned:

(1) Identification of crucial moments. The moment by moment recording of EDA activity makes it possible to identify special segments in the ongoing interaction characterized by atypical, augmented or attenuated activity and to analyze if these segments are associated with specific communication behaviors. Of course, the analysis may proceed the other way around: identifying EDA patterns associated with specific communication content.

(2) Physiological markers of interpersonal synchrony. A fascinating aspect of the psychophysiology of interpersonal interaction is the synchrony in physiological response between the two parties involved. Marci et al. found that high concordance between EDA activity of therapist and patient during 
Finset, A., Stensrud, T.L., Holt, E., Verheul, W., Bensing, J. Electrodermal activity in response to empathic statements in clinical interviews with fibromyalgia patients. Patient Education and Counseling: 2011, 82(3) $355-360$

psychotherapy was associated with more positive social-emotional interactions [14]. Physiological synchrony has also been extensively studied in married couples [28] and [29].

\subsection{Conclusion}

In this study, empathic statements by the interviewer were associated with increased SCL in patients. The study illustrates the potential relevance of psychophysiological methods in clinical communication research. Combining data from behavioral observation and psychophysiology may enhance the exploration of interplay between conscious and unconscious processes [12] and lead to a better insight into the dynamics of emotion in medical interviews, with potential implications for the communication behavior of clinicians. However, the limitations of this approach should also be recognized. Since EDA not always may be validated by subjective experience, it is sometimes difficult to interpret findings. Increased values should not automatically be considered as an indicator of stress or negative emotions, and different psychophysiological measures should be applied. Individual differences must be taken into account when data are analyzed.

\subsection{Practice implications}

Psychophysiological variables such as EDA may be applied in clinical communication research, as biological markers of components of emotion. Clinicians should be aware of the finding that empathic statements in clinical interviews may be associated with increased psychophysiological arousal.

\section{Conflict of interest}

None declared.

\section{Acknowledgements}

The authors wish to thank the patients who participated in the study and Dr. Sigrid Hørven Wigers for kind assistance during data collection.

\section{REFERENCES}

[1] C. Zimmermann, L. Del Piccolo and A. Finset, Cues and concerns by patients in medical consultations: a literature review, Psychol Bull 133 (2007), pp. 438-463.

[2] P.N. Butow, R.F. Brown, S. Cogar, M.H.N. Tattersall and S.M. Dunn, Oncologists' reactions to cancer patients' verbal cues, Psycho-Oncol 11 (2002), pp. 47-58.

[3] K.A. Stepien and A. Baernstein, Educating for empathy: a review, J Gen Int Med 21 (2006), pp. 524530. View Record in Scopus | Cited By in Scopus (36)

[4] R. Pedersen, Empirical research on empathy in medicine - a critical review, Patient Educ Counsel 76 (2009), pp. 307-322.

[5] H.M. Adler, The so ciophysiology of caring in the doctor-patient relationship, J Gen Int Med 17 (2002), pp. 883-890.

[6] M.E. Dawson, A.M. Schell and D.L. Filion, The electrodermal system. In: J.T. Cacioppo, L.G. Tassinary and G.G. Berntson, Editors, Handbook of psychophysiology, Cambridge University Press, Cambridge (2007), pp. 159-181.

[7] M.M. Bradley, M. Codispoti, D. Sabatinelli and P.J. Lang, Emotion and motivation II: sex differences in picture processing, Emotion 1 (2001), pp. 300-319.

[8] S.A. Shields, K.A. MacDowell, S.B. Fairchild and M.L. Campbell, Is mediation of sweating cholinergic, adrenergic, or both? A comment on the literature, Psychophysiology 24 (1987), pp. 312-319.

[9] S.D. Kreibig, Autonomic nervous system activity in emotion: a review, Biol Psychol 84 (2010), pp. 394 421.

[10] $\mathrm{H}$. Storm, Changes in skin conductance as a tool to monitor nociceptive stimulation and pain, Curr Opin Anaesthesiol 21 (2008), pp. 796-804.

[11] R. Hoehn-Saric, D.R. McLeod, F. Funderburk and P. Kowalski, Somatic symptoms and physiologic responses in generalized anxiety disorder and panic disorder: an ambulatory monitor study, Arch Gen Psychiat 61 (2004), pp. 913-921.

[12] C. Marci and H. Riess, Support for the psychobiology of empathy and psychodynamic process. The clinical relevance of psychophysiology, Am J Psychother 59 (2005), pp. 213-226. View Record in Scopus I Cited By in Scopus (3) 
Finset, A., Stensrud, T.L., Holt, E., Verheul, W., Bensing, J. Electrodermal activity in response to empathic statements in clinical interviews with fibromyalgia patients. Patient Education and Counseling: 2011, 82(3) $355-360$

[13] C.D. Marci and S.P. Orr, The effect of emotional distance on psychophysiologic concordance and perceived empathy between patient and interviewer, Appl Psychophysiol Biofeedback 31 (2006), pp. 115128.

[14] C.D. Marci, J. Ham, E. Moran and S.P. Orr, Physiologic correlates of perceived therapist empathy and social-emotional process during psychotherapy, J Nerv Ment Dis 195 (2007), pp. 103-111.

[15] L. Ellington, S. Matwin, B.N. Uchino, S. Jasti, W.N. Dudley and D. Roter, The body's response to health care provider communication: the impact of dominant versus facilitative styles, J Appl Biobehav Res 13 (2008), pp. 67-85.

[16] A.L. Suchman, K. Markakis, H.B. Beckman and R. Frankel, A model of empathic communication in the medical interview, J Am Med Assoc 277 (1997), pp. 678-682. View Record in Scopus | Cited By in Scopus (243)

[17] S.J. Guastello, D. Pincus and P.R. Gunderson, Electrodermal arousal between participants in a conversation: nonlinear dynamics and linkage effects, Nonlin Dynam Psychol Life Sci 10 (2006), pp. 365399. View Record in Scopus | Cited By in Scopus (5)

[18] F. Wolfe, D.J. Clauw, M.-A. Fitzcharles, D.L. Goldenberg, R.S. Katz, P. Mease, A.S. Russell, I.J. Russell, J.B. Winfield and M.B. Yunus, The American College of Rheumatology preliminary diagnostic criteria for fibromyalgia and measurement of symptom severity, Arthritis Care Res 62 (2010), pp. 600610.

[19] A. Werner and K. Malterud, It is hard work behaving as a credible patient: encounters between women with chronic pain and their doctors, Soc Sci Med 57 (2003), pp. 1409-1419.

[20] L. Haugli, E. Strand and A. Finset, How do patients with rheumatic disease experience their relationship with their doctors? A qualitative study of experiences of stress and support in the doctorpatient relationship, Patient Educ Counsel 52 (2004), pp. 169-174.

[21] K. Thieme, U. Rose, T. Pinkpank, C. Spies, D.C. Turk and H. Flor, Psychophysiological responses in patients with fibromyalgia syndrome, J Psychosom Res 61 (2006), pp. 671-679.

[22] N. Christenfeld and W. Gerin, Social support and cardiovascular reactivity, Biomed Pharmacother 54 (2000), pp. 251-257.

[23] D. Watson, L.A. Clark and A. Tellegen, Development and validation of brief measures of positive and negative affect: the PANAS scales, J Pers Soc Psychol 54 (1988), pp. 1063-1070.

[24] S. Steine, A. Finset and E. Laerum, A new, brief questionnaire (PEQ) developed in primary health care for measuring patients' experience of interaction, emotion and consultation outcome, Fam Pract 18 (2001), pp. 410-418.

[25] J.J. Gross, Antecedent- and response-focused emotion regulation: divergent consequences for experience, expression, and physiology, J Pers Soc Psychol 74 (1998), pp. 224-237.

[26] E. Lush, P. Salmon, A. Floyd, J.L. Studts, I. Weissbecker and S.E. Sephton, Mindfulness meditation for symptom reduction in Fibromyalgia: psychophysiological correlates, J Clin Psychol Med Settings 16 (2009), pp. 200-207.

[27] G. Stemmler and J. Wacker, Personality, emotion, and individual differences in physiological responses, Biol Psychol 84 (2010), pp. 541-551. [28] R.W. Levenson and J.M. Gottman, Marital interaction: physiological linkage and affective exchange, J Pers Soc Psychol 45 (1983), pp. 587-597.

[29] R.W. Levenson and J.M. Gottman, Physiological and affective predictors of change in relationship satisfaction, J Pers Soc Psychol 49 (1985), pp. 85-94.

\section{[TABLES AND FIGURES]}


Finset, A., Stensrud, T.L., Holt, E., Verheul, W., Bensing, J. Electrodermal activity in response to empathic statements in clinical interviews with fibromyalgia patients. Patient Education and Counseling: 2011, 82(3) 355-360

Table 1

Effects of experimental conditions on SCL and patient satisfaction. Two-ways ANOVA.

\begin{tabular}{|c|c|c|c|c|c|c|}
\hline \multirow[b]{2}{*}{ Independent variables } & \multicolumn{2}{|c|}{$\mathrm{SCL}$ at $\mathrm{T} 2$} & \multicolumn{2}{|c|}{ SCL at T3 } & \multicolumn{2}{|c|}{ Satisfaction } \\
\hline & $M$ & SD & $M$ & SD & $M$ & SD \\
\hline \multicolumn{7}{|l|}{ Empathy condition } \\
\hline Emotional content & & & 5.35 & 4.47 & 90.40 & 4.79 \\
\hline Neutral content & & & 2.57 & .91 & 85.67 & 8.72 \\
\hline \multicolumn{6}{|l|}{ Inattention condition } & 7.45 \\
\hline Emotional content & & & 3.07 & 1.90 & 76.45 & 16.79 \\
\hline Neutral content & & & 3.25 & 2.92 & 73.27 & 7.21 \\
\hline Inattention, total & 2.82 & 2.19 & 3.16 & 2.41 & 74.86 & 12.86 \\
\hline All patients & 3.12 & 2.75 & 3.46 & 2.85 & 81.34 & 12.28 \\
\hline \multicolumn{7}{|l|}{ Analysis of variance } \\
\hline \multirow[t]{2}{*}{ Main effect of Empathy vs. Inattention } & \multirow{2}{*}{\multicolumn{2}{|c|}{$F=5.21 ; p<.05$}} & \multirow{2}{*}{\multicolumn{2}{|c|}{$F=6.28 ; p<.01$}} & \multirow{2}{*}{\multicolumn{2}{|c|}{$F=17.05 ; p<.0001$}} \\
\hline & & & & & & \\
\hline \multicolumn{3}{|l|}{ Main effect of Emotional vs. Neutral } & \multirow{2}{*}{\multicolumn{2}{|c|}{$F=.13 ;$ N.s. }} & \multicolumn{2}{|c|}{$\begin{array}{l}\text { Partial ta sq. }=.30 \\
F=1.54 ; \text { N.s. }\end{array}$} \\
\hline & & & & & \multicolumn{2}{|c|}{ Partial Eta Sq. $=.04$} \\
\hline \multirow[t]{2}{*}{ Interaction effect: } & & & \multicolumn{2}{|c|}{$F=7.27 ;<.01$} & \multirow{2}{*}{\multicolumn{2}{|c|}{$\begin{array}{l}F=.06 ; \text { N.s. } \\
\text { Patio }\end{array}$}} \\
\hline & & & \multicolumn{2}{|c|}{ Partial Eta Sq. $=.18$} & & \\
\hline
\end{tabular}

SCL

Scenario A

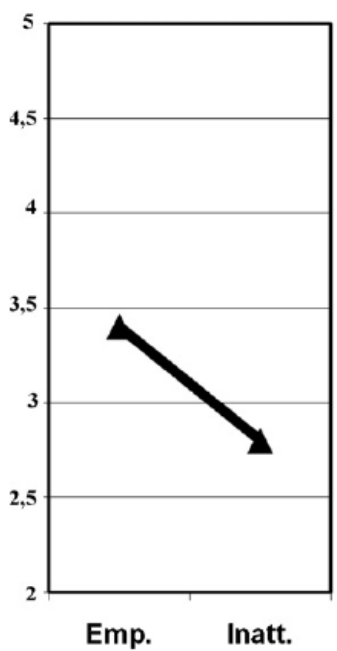

SCL

Scenario B

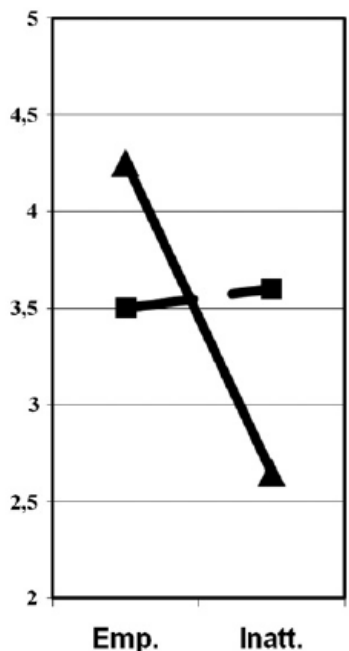

Satisfaction

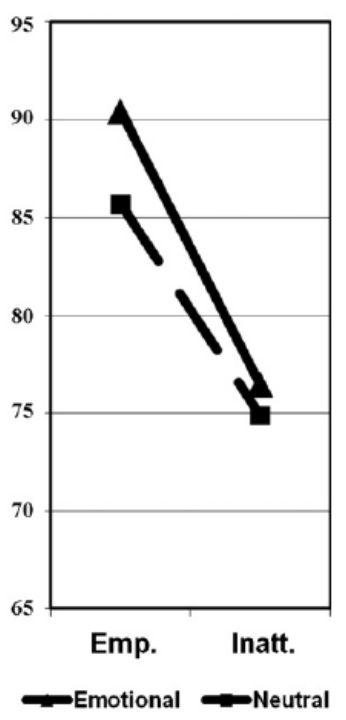

Fig. 1. SCL level during scenarios A and B respectively of the experiment, controlling for SCL at baseline (estimated marginal means) and patient satisfaction scores broken down on experimental conditions (Empathy vs. Inattention to emotion and Emotional vs. Neutral content). 